Artículo

\title{
Potencial de secuestro de carbono orgánico en quinua simulado con el modelo RothC-26.3
}

\author{
Lucila González Molina ${ }^{1 \S}$ \\ Eduardo Espitia-Rangel ${ }^{1}$ \\ Joel Pineda-Pineda ${ }^{2}$ \\ Erica Muñiz Reyes ${ }^{1}$ \\ Martha G. Irizar Garza ${ }^{1}$ \\ Alma Ayala Garay ${ }^{1}$
}

${ }^{1}$ Campo Experimental Valle de México-INIFAP. Carretera Los Reyes-Texcoco km 13.5, Coatlinchán, Estado de México. CP. 56250. (espitia.eduardo@inifap.gob.mx; muniz.erica@inifap.gob.mx; irizar.martha@inifap.gob.mx; ayala.alma@nifap.gob.mx). ${ }^{2}$ Universidad Autónoma Chapingo. Carretera México-Texcoco km 38.5, Texcoco, Estado de México. CP. 56230. (pinedapjoel@yahoo.com.mx).

${ }^{\S}$ Autora para correspondencia: gonzalez.lucila@inifap.gob.mx.

\section{Resumen}

El presente estudio se realizó en el Campo Experimental Valle de México de INIFAP con el objetivo de estimar el potencial secuestro de carbono orgánico del suelo (COS) en las variedades de quinua: Amarilla Maranganí y Blanca con el uso del modelo RothC-26.3. Las simulaciones de la dinámica del COS con el RothC incluyeron: tres periodos de tiempo: 20, 60 y 100 años, los sistemas anuales: monocultivo de quinua (MQ), monocultivo de maíz (MM) y rotación de quinuamaíz (RQM); y el uso de tres aportes de carbono (C) al suelo de los residuos vegetales de cosecha (RV): 60,70 y $80 \%$ de la materia seca total (MST). Al considerar que cerca de $80 \%$ de la MST queda sobre el terreno de cultivo después de la cosecha de quinua, la acumulación de carbono (C) se evaluó por estructura vegetal en tres en tratamientos de fertilización. La fertilización no tuvo efecto significativo en la producción de la MST, atribuido al nivel favorable de fertilidad del suelo del sitio de estudio y a la rusticidad del cultivo. La MST de Amarilla Maranganí fue mayor a la de Blanca. En ambas quinuas, los tallos y las inflorescencias y las hojas y el grano, representaron del 76 a $84 \%$ y del 11 a $23 \%$ de la MST, respectivamente. Los cambios de COS simulados por el RothC en ambas variedades de quinua indicaron potencial de secuestro de $\operatorname{COS}\left(\mathrm{Mg} \mathrm{C} \mathrm{ha}^{-1} \mathrm{año}^{-1}\right)$ en un periodo de tiempo mayor a 20 años, sólo en el sistema MQ con RV=80\% MST.

Palabras clave: carbono edáfico; residuos de vegetales; sistemas de cultivo, tasa de cambio.

Recibido: marzo de 2020

Aceptado: mayo de 2020 


\section{Introducción}

Los suelos agrícolas tienen un potencial significativo para secuestrar carbono orgánico del suelo (COS) y mitigar la emisión de gases de efecto invernadero (Zhang et al., 2014; Paustian et al., 2016). A nivel mundial, los suelos agrícolas han perdido entre 30 y $75 \%$ de las reservas de COS (30 a $40 \mathrm{Mg} \mathrm{C} \mathrm{ha}^{-1}$ ) (Lal et al., 2007). Sin embargo, 75\% de estos suelos, pueden recuperarse a través del incremento del COS almacenado o secuestrado. Según Lal (2004), el COS almacenado depende de las estrategias de manejo como la retención de residuos y aplicación de fertilizantes, además de las condiciones ambientales (Luo et al., 2010).

El modelo RothC-26.3 es uno de los más usados en la predicción de los cambios del C en el suelo y se ha aplicado en diferentes sistemas y suelos del mundo, además de la disponibilidad y simplicidad de los datos de entrada para ejecutarlo (Coleman et al., 1997). En México, el desempeño del RothC-26.3 se ha evaluado en experimentos de corta duración, con el uso de mediciones directas en sistemas agrícolas, forestales, pastizales y agostaderos (González et al., 2011). En los sistemas agrícolas se reportó una eficiencia del de 0.78 a 0.87 (González et al., 2011) y de 0.77 (González et al., 2017).

En los cultivos denominados pseudocereales como la quinua, amaranto y chía, la información del secuestro de COS por el aporte de los residuos vegetales de cosecha es nula. En el caso de la quinua tiene potencial de almacenar COS en el suelo ya que al igual que el cultivo de amaranto, en México, después de su cosecha, se dejan alrededor de $80 \%$ de los residuos de vegetales aéreos en los terrenos de cultivo, mientras que el maíz, bajo labranza convencional, según las estimaciones de Pérez et al. (2000) de 15 a $30 \%$ de RV.

El objetivo del presente trabajo fue estimar el potencial de secuestro de carbono orgánico del suelo de sistemas de cultivo de quinua con el modelo RothC-26.3. Se generaron escenarios de simulación en periodos de tiempo de 20,60 y 100 años y con diferentes aportes de residuos de cosecha en los sistemas de cultivo anual: monocultivos de maíz y quinua; y rotación de quinua-maíz.

\section{Materiales y métodos}

\section{Características del sitio experimental}

El estudio se realizó en el Campo Experimental Valle de México del INIFAP, ubicado, en la localidad de Santa Lucía, Coatlinchán, Estado de México. Este sitio se caracteriza por su clima templado frío con lluvias en verano, como se puede observar en el Cuadro 1, donde también se indican la localización geográfica y las características climáticas y edáficas.

Cuadro 1. Localización geográfica y características climáticas y edáficas del sitio experimental.

\begin{tabular}{cc}
\hline Localización & Características \\
\hline Longitud & $98^{\circ} 53^{\prime} \mathrm{W}$ \\
Latitud & $19^{\circ} 29^{\prime} \mathrm{N}$ \\
Altitud $(\mathrm{m})$ & 2240 \\
Temperatura media anual ${ }^{8}\left({ }^{\circ} \mathrm{C}\right)$ & 18.6 \\
\hline
\end{tabular}




\begin{tabular}{cc}
\hline Localización & Características \\
\hline Precipitación media anual $^{\S}(\mathrm{mm})$ & 590 \\
Evaporación media anual $^{\S}(\mathrm{mm})$ & 54.7 \\
Suelo $^{\epsilon}$ & Feozem \\
Textura & Franco arcillosa \\
Arcilla $(\%)$ & 38 \\
Densidad aparente $\left(\mathrm{g} \mathrm{cm}^{-3}\right)$ & 0.87 \\
pH & 7 \\
Materia orgánica $(\%)$ & 2.3 \\
$\mathrm{~N}-\mathrm{NH}_{4}(\mathrm{ppm})$ & 21 \\
$\mathrm{~N}-\mathrm{NO}_{3}(\mathrm{ppm})$ & 70 \\
P extractable Olsen $(\mathrm{ppm})$ & 55 \\
\hline
\end{tabular}

${ }^{8}=$ Datos de la estación Chapingo de 1995-2016; ${ }^{\epsilon}=$ INEGI (2007).

\section{Determinación de COS}

La determinación del COS se llevó a cabo antes del establecimiento del experimento en el mes de junio de 2016. Se tomaron seis muestras de suelo distribuidas en la superficie experimental de 240 $\mathrm{m}^{2}$, en la profundidad de suelo $(\mathrm{Pm})$ de $0-30 \mathrm{~cm}$ con el siguiente procedimiento: las muestras de suelo se secaron al aire, se registró su peso; con auxilio de pinzas se retiraron raíces, restos vegetales y animales visibles; se tamizaron a $2 \mathrm{~mm}$; y se tomaron submuestras. La determinación de la concentración de COS se realizó con el método de Walkley y Black (1934) que reporta la materia orgánica del suelo (MOS) y se utilizó el factor 0.58 (1/1.724) para convertir MOS a COS.

Para el cálculo de COS $\left(\mathrm{Mg} \mathrm{ha}^{-1} \mathrm{año}^{-1}\right)$, se obtuvo la densidad aparente (Da) $\left(\mathrm{g} \mathrm{cm}^{-3}\right)$ por el método del cilindro. La Da fue la relación entre el peso del suelo seco y el volumen del suelo: el peso de suelo mineral más el humificado, sin considerar el peso de las raíces, residuos y piedras y el volumen del suelo, se calculó al descontar del volumen total, el volumen de raíces, residuos y piedras. La cantidad de COS $\left(\mathrm{Mg} \mathrm{ha}^{-1}\right)$ fue el producto de Pm, Da y el\% COS.

\section{Aporte de carbono al suelo por residuos vegetales}

\section{Quinua}

Al considerar que cerca de $80 \%$ de la MST de quinua queda en el terreno de cultivo después de la cosecha, se evaluó la acumulación de carbono (C) en la materia seca por estructura vegetal y total en tres en tratamientos de dosis de fertilización y un testigo. Según Bazile et al. (2014), en la cosecha en forma manual o mecánica, después de cortar las panojas o inflorescencias, estas se dejan en el campo agrupadas en montones para que se sequen completamente.

Posteriormente se procede a la trilla, sobre una lona se colocan las panojas, con ayuda de un implemento agrícola o golpeándolas con una vara se desprende el grano, de esta manera, la mayor parte de los residuos de cosecha quedan en los terrenos de cultivo. Los tratamientos de dosis de fertilización fueron los siguientes: T1=40-40-00; T2=80-40-00; T3=120-40-00 y T0=00-00-00. Las unidades experimentales fueron de cinco surcos de $0.8 \mathrm{~m}$ de ancho por $5 \mathrm{~m}$ de largo $\left(20 \mathrm{~m}^{2}\right)$. 
Estos tratamientos se evaluaron en un diseño experimental en bloques al azar con tres repeticiones en arreglo de parcelas divididas. En las parcelas se establecieron las variedades de quinua Amarilla Maranganí y Blanca y en los bloques los tratamientos de dosis de fertilización, en total hubo 24 unidades experimentales $\left(240 \mathrm{~m}^{2}\right)$. Además de los $\mathrm{RV}$, el aporte de $\mathrm{C}$ al suelo se dio por el abono que se colocó sobre el suelo junto con la semilla al momento de la siembra.

La materia seca se midió cuando el cultivo alcanzó la madurez fisiológica a los 147 días después de la siembra y consistió de las siguientes actividades: se cortaron cuatro plantas al ras del suelo, se separaron las estructuras vegetales: hojas, tallos, inflorescencias y grano, se obtuvo el peso fresco total y el peso en seco de una submuestra de 100-200 g, la submuestra fue puesta a secar en una estufa a una temperatura de $65^{\circ} \mathrm{C}$. Con estos últimos datos se obtuvo la materia seca por órgano al substraer el porcentaje de humedad del peso de la biomasa total en fresco.

El porcentaje de humedad fue el peso húmedo menos el peso seco entre el peso húmedo de las submuestras. La biomasa de las raíces fue estimada como un 10\% de la biomasa aérea total, al considerar que en otros cereales como trigo y cebada es 10 a $15 \%$ según el estudio de Kuzyakov y Domanski (2000). El carbono por órgano fue el producto de la materia seca (g) y la concentración de carbono promedio de tres repeticiones en el tejido vegetal (\%). La concentración de carbono en el tejido vegetal se determinó por combustión seca en un analizador automático de carbono orgánico total (Shimadzu TOC 5000-A).

\section{Variables de crecimiento y rendimiento}

Para comparar el crecimiento y rendimiento de las variedades de quinua en estudio, en la etapa de madurez fisiológica se midieron las siguientes variables: altura de planta, medida desde la base del tallo a la punta de la panoja principal, diámetro de tallo, medida a $10 \mathrm{~cm}$ de la base de la planta, longitud de panoja, materia seca total, rendimiento de grano por planta y por hectárea e índice de cosecha (IC).

\section{Maíz}

En la estimación de los RV que aporta el maíz al suelo, se consideró la información para la zona de mediana productividad bajo temporal estricto de Texcoco que es similar a las condiciones bajo las cuales se evaluó el cultivo de quinua. Se consideró un rendimiento de $3 \mathrm{Mg} \mathrm{ha}^{-1}$ según la SAGARPA (2015) y un índice de cosecha de 0.34. Esta información dio una entrada anual de RV de $3.5 \mathrm{Mg} \mathrm{C}^{-1}$ año $^{-1}$ que corresponde a $40 \%$ de la materia seca total, $10 \%$ lo aportan las raíces de acuerdo con las estimaciones de Kuzyakov y Domanski (2000) para cereales y 30\% los tallos de maíz (Pérez et al., 2000).

\section{Modelo RothC}

El modelo RothC de Coleman y Jenkinson (2005) divide el COS en compartimentos que contienen materiales con diferentes tasas de descomposición, cuatro son activos y uno es pasivo: (i) material vegetal de fácil descomposición (MVF); (ii) material vegetal resistente (MVR); (iii) biomasa microbiana (BIO); (iv) materia orgánica humificada (HUM); y (v) materia orgánica inerte (MOI). 
Los compartimentos activos experimentan descomposición por cinética de primer orden, según la siguiente expresión: $\mathrm{Y}=\mathrm{Y}_{0}\left(1-\mathrm{e}^{\text {-abckt}}\right)$. Donde: $\mathrm{Y}_{0}$ es el $\mathrm{C}$ inicial del compartimento activo; $\mathrm{k}$ es la tasa constante de descomposición anual; $\mathrm{t}$ es 1/12 para obtener la tasa de descomposición al final de cada mes; y a, b y c son los factores que modifican a $\mathrm{k}$ y son temperatura, humedad y cobertura del suelo, respectivamente. El valor de k para cada compartimento presenta los siguientes valores: MVD (10), MVR (0.3), BIO (0.66) y HUM (0.2).

El compartimento pasivo, MOI $\left(\mathrm{Mg} \mathrm{ha}^{-1}\right)$ se obtuvo con la ecuación de Falloon et al. (1998) expresada por MOI $=0.049 \times \mathrm{COT}^{1.139}$, donde COT es el carbono orgánico $\left(\mathrm{Mg} \mathrm{ha}^{-1}\right)$. Los datos de entrada del modelo RothC son: 1) factores climáticos: promedio mensual de temperatura del aire $\left({ }^{\circ} \mathrm{C}\right)$, precipitación $(\mathrm{mm})$ y evaporación $(\mathrm{mm})$, TPM, PPM y EPM, respectivamente; 2) edáficos: contenido de $\operatorname{COS}\left(\mathrm{Mg} \mathrm{ha}^{-1}\right)$, contenido de arcilla (\%) y profundidad de muestreo del suelo; 3 ) entrada mensual de $\mathrm{C}$ al suelo de residuos vegetales (RV) y abonos orgánicos (AO); 4) cobertura vegetal mensual del suelo; es decir, si el suelo está desnudo o con cobertura vegetal; 5) la relación MVD/MVR, que de acuerdo con Coleman y Jenkinson (2005) fue de 1.44 (59\% son para MVD y $41 \%$ son para MVR); y 6) el valor de la MOI obtenido de la ecuación de Falloon et al. (1998).

\section{Simulaciones con el modelo RothC}

La simulación de los cambios de COS incluyó la inicialización y elaboración de escenarios. En la inicialización, se obtuvo el contenido de $\mathrm{C}$ inicial de los compartimentos activos en la condición del suelo en equilibrio. Esto se logró ejecutando el RothC iterativamente 10000 años con la información de clima, suelo, relación MVD/MVR, MOI y entrada de $\mathrm{C}$ de los RV al suelo obtenidos por el modelo. Los escenarios, se ejecutaron con el valor de $\mathrm{C}$ de los compartimentos activos y pasivo, que se obtuvieron en la inicialización y con la información de clima, suelo, MVD/MVR, cobertura del suelo y MOI.

Las simulaciones de los escenarios de la dinámica del COS con el RothC se efectuaron con la información: tres periodos de tiempo: 20, 60 y 100 años; los sistemas anuales: monocultivo de quinua (MQ), monocultivo de maíz (MM), rotación de quinua-maíz (RQM) y el uso de aporte de C al suelo proveniente de los RV: 60, 70 y $80 \%$ de la MST de quinua y 40\% de la MST de maíz.

El aporte de $\mathrm{C}$ por hectárea $\left(\mathrm{Mg} \mathrm{ha}^{-1} \mathrm{año}^{-1}\right)$ en quinua se calculó como el producto de la proporción de la MST (\%) y la densidad de población (plantas ha ${ }^{-1}$ ): 129, 166 y 136, 458 para Amarilla Maranganí y Blanca, respectivamente. Para determinar la tasa de cambio (TC) del COS en los periodos de tiempo 20, 40 y 60 años, se usó la siguiente expresión: $\operatorname{TC~}^{C_{0 S}} 20$ años $=\operatorname{COS}_{\text {año } 2000}$ -

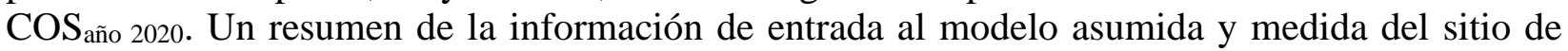
estudio se muestra en los Cuadros 1,2 y 3 .

Cuadro 2. Información necesaria en la inicialización y simulación de escenarios en variedades de quinua con el modelo RothC.

\begin{tabular}{cccccccc}
\hline \multirow{2}{*}{ Variedades } & \multicolumn{2}{c}{ COS } & MOI & & arcilla & CVS & \multirow{2}{*}{ MVD/MVR } \\
\cline { 2 - 3 } & \multicolumn{2}{c}{$\left(\mathrm{Mg} \mathrm{ha}^{-1}\right)$} & & $(\%)$ & (meses $)$ & \\
\hline Amarilla Maranganí & 34.7 & 2.78 & & 38 & 8 & 1.44 \\
Blanca & 34.7 & 2.78 & & 38 & 8 & 1.44 \\
Maíz & 34.7 & 2.78 & & 38 & 8 & 1.44 \\
\hline
\end{tabular}


Cuadro 3. Entrada de carbono al suelo por los residuos vegetales en la inicialización y simulación de escenarios en variedades de quinua con el modelo RothC.

\begin{tabular}{|c|c|c|c|c|c|c|}
\hline \multicolumn{2}{|c|}{ Inicialización } & \multicolumn{4}{|c|}{ Escenarios RV (\% de materia seca total) } & \multirow{2}{*}{ Abono } \\
\hline Variedades & $\mathrm{RV}_{\text {RothC }}$ & 40 & 60 & 70 & 80 & \\
\hline & & \multicolumn{5}{|c|}{$\left(\mathrm{Mg} \mathrm{C} \mathrm{ha-1} \mathrm{año}^{-1}\right)$} \\
\hline Amarilla Maranganí & 4.77 & & 3.84 & 4.48 & 5.12 & 0.24 \\
\hline Blanca & 4.77 & & 3.52 & 4.08 & 4.72 & 0.24 \\
\hline Maíz & 4.77 & 3.5 & & & & 0 \\
\hline
\end{tabular}

$\mathrm{RV}=$ residuos vegetales.

\section{Análisis estadístico de las variables de estudio}

La acumulación de $\mathrm{C}$ en la materia seca por estructura vegetal y total, y las variables de crecimiento y de rendimiento se sometieron a un análisis de varianza con el procedimiento LGM de SAS. Para comparación de medias se usó la prueba de Tukey $(p=0.05)$.

\section{Resultados y discusión}

\section{Distribución de carbono en la biomasa}

Los resultados de la comparación de medias de las variables de crecimiento y rendimiento para las quinuas Amarilla Maranganí y Blanca (Cuadro 4), indicaron diferencias estadísticas para altura de planta, diámetro de tallo, materia seca total y rendimiento de grano por hectárea, en todos los casos la Amarilla Maranganí fue superior a la Blanca.

Cuadro 4. Comparación de medias para variables de crecimiento y rendimiento en las quinuas de estudio.

\begin{tabular}{cccccccc}
\hline Quinua/Variables & $\begin{array}{c}\text { Alt } \\
(\mathrm{cm})\end{array}$ & $\begin{array}{c}\mathrm{D} \\
(\mathrm{cm})\end{array}$ & $\begin{array}{c}\mathrm{LP} \\
(\mathrm{cm})\end{array}$ & $\begin{array}{c}\text { Rendimiento } \\
\left(\mathrm{kg} \mathrm{ha}^{-1}\right)\end{array}$ & $\begin{array}{c}\text { RP } \\
\left(\mathrm{g} \mathrm{planta}^{-1}\right)\end{array}$ & $\begin{array}{c}\text { MST } \\
\left(\mathrm{g} \mathrm{planta}^{-1}\right)\end{array}$ & IC \\
\hline Amarilla Maranganí & $214 \mathrm{a}$ & $15 \mathrm{a}$ & $74 \mathrm{a}$ & $2509 \mathrm{a}$ & $18.2 \mathrm{a}$ & $109 \mathrm{a}$ & $0.18 \mathrm{a}$ \\
Blanca & $149 \mathrm{~b}$ & $13 \mathrm{~b}$ & $72 \mathrm{~b}$ & $1172 \mathrm{~b}$ & $17.6 \mathrm{a}$ & $94 \mathrm{a}$ & $0.17 \mathrm{a}$ \\
DMSH & 13.66 & 0.57 & 0.62 & 472.24 & 6.7 & 35.29 & 0.032 \\
\hline
\end{tabular}

Alt= altura de planta; $\mathrm{D}=$ diámetro; $\mathrm{LP}=$ longitud de panoja; $\mathrm{RP}=$ rendimiento por planta; $\mathrm{MST}=$ materia seca total; $\mathrm{IC}=$ índice de cosecha; DMSH= diferencia mínima significativa honesta. Valores que comparten la misma letra en la misma columna son estadísticamente iguales (Tukey, $p=0.05$ ).

El rendimiento por hectárea en Amarilla Mananganí fue cercano al promedio nacional de $2.8 \mathrm{Mg}$ $\mathrm{ha}^{-1} \mathrm{y}$ en ambas fue alto en el tratamiento de fertilización T3 (120-40-00), aunque no presentó diferencias significativas entre tratamientos de fertilización. La longitud de panoja estuvo dentro del rango de 30 a $80 \mathrm{~cm}$, reportado por Mujica et. al. (2003) para las quinuas peruanas. El IC obtenido en el presente estudio fue menor al rango promedio reportado en otros estudios, Mujica et al. (2003) quien reportó un rango en quinua de 0.24 a 0.45 . 
El rendimiento $\left(\mathrm{kg} \mathrm{ha}^{-1}\right)$ del presente estudio fue superior al promedio reportado experimentalmente por Mujica et al. (2003) en las mismas variedades cultivadas de Perú (0.93 kg ha-1) y también fue superior para el caso de Amarilla cultivada en Viacollo, Chile donde presentó una producción de $1.35 \mathrm{~kg} \mathrm{ha}^{-1}$ (Delatorre et al., 2008).

\section{Aporte de carbono vegetal al suelo}

Las medias de la acumulación de $\mathrm{C}$ en hoja, inflorescencia, y grano, MST e IC fueron estadísticamente iguales en ambas quinuas, la excepción se presentó en los tallos, con mayor valor en Amarilla Maranganí (Cuadro 5).

Cuadro 5. Comparación de medias de contenido de carbono por órgano por planta en las quinuas.

\begin{tabular}{lcccccc}
\hline \multirow{2}{*}{ Quinua/Variables } & Tallo & Hoja & Inflorescencia & Grano & MST & \multirow{2}{*}{ IC } \\
\cline { 2 - 6 } & \multicolumn{7}{c}{$\left(\mathrm{g} \mathrm{planta}^{-1}\right)$} \\
\hline Amarilla Maranganí & $20 \mathrm{a}$ & $0.79 \mathrm{a}$ & $16 \mathrm{a}$ & $8.2 \mathrm{a}$ & $45 \mathrm{a}$ & $0.18 \mathrm{a}$ \\
Blanca & $14 \mathrm{~b}$ & $0.43 \mathrm{a}$ & $17 \mathrm{a}$ & $7.9 \mathrm{a}$ & $39 \mathrm{a}$ & $0.2 \mathrm{a}$ \\
DMSH & 6.04 & 0.5 & 7.35 & 1.29 & 14.55 & 0.043 \\
\hline
\end{tabular}

MST= materia seca total; $\mathrm{IC}=$ índice de cosecha; $\mathrm{DMSH}=$ diferencia mínima significativa honesta. Valores que comparten la misma letra en la misma columna son estadísticamente iguales (Tukey, $p=0.05$ ).

El Cuadro 6, muestra el aporte de C al suelo por las quinuas y el maíz. La distribución de C por órgano: tallo, hoja, inflorescencia, grano y raíz, en los tratamientos de fertilización en quinua no presentó diferencias significativas a pesar de esto, el T3 (120-40-00) tuvo la mayor cantidad de C por órgano y MST total.

Cuadro 6. Carbono en residuos vegetales de quinua (g) Amarilla Maranganí, Blanca y de maíz en madurez fisiológica.

\begin{tabular}{|c|c|c|c|c|}
\hline Órgano/tratamientos fertilización & T0 & $\mathrm{T} 1$ & $\mathrm{~T} 2$ & $\mathrm{~T} 3$ \\
\hline \multicolumn{5}{|c|}{ Amarilla Maranganí } \\
\hline Tallo & $18.45 \mathrm{a}$ & $19.24 \mathrm{a}$ & $18.71 \mathrm{a}$ & $21.99 \mathrm{a}$ \\
\hline Hoja & $0.42 \mathrm{a}$ & $0.71 \mathrm{a}$ & $1.12 \mathrm{a}$ & $0.91 \mathrm{a}$ \\
\hline Inflorescencia & $17.11 \mathrm{a}$ & $15.76 \mathrm{a}$ & $13.91 \mathrm{a}$ & $18.97 \mathrm{a}$ \\
\hline Grano & $8.97 \mathrm{a}$ & $7.68 \mathrm{a}$ & $6.57 \mathrm{a}$ & $9.46 \mathrm{a}$ \\
\hline Raíces & $4.5 \mathrm{a}$ & $4.5 \mathrm{a}$ & $4 \mathrm{a}$ & $5.1 \mathrm{a}$ \\
\hline MST & $49.4 \mathrm{a}$ & $47.7 \mathrm{a}$ & $44.3 \mathrm{a}$ & $56.5 \mathrm{a}$ \\
\hline \multicolumn{5}{|c|}{ Blanca } \\
\hline Tallo & $13.55 \mathrm{a}$ & $13.2 \mathrm{a}$ & $12.37 \mathrm{a}$ & $13.8 \mathrm{a}$ \\
\hline Hoja & $0.33 \mathrm{a}$ & $0.46 \mathrm{a}$ & $0.48 \mathrm{a}$ & $0.44 \mathrm{a}$ \\
\hline Inflorescencia & $17.75 \mathrm{a}$ & $17.15 \mathrm{a}$ & $15.12 \mathrm{a}$ & $19.44 \mathrm{a}$ \\
\hline Grano & $9.17 \mathrm{a}$ & $7.57 \mathrm{a}$ & $6.29 \mathrm{a}$ & $8.53 \mathrm{a}$ \\
\hline Raíces & $4.1 \mathrm{a}$ & $3.8 \mathrm{a}$ & $3.4 \mathrm{a}$ & $4.2 \mathrm{a}$ \\
\hline MST & $44.9 \mathrm{a}$ & $42.2 \mathrm{a}$ & $37.7 \mathrm{a}$ & $46.4 \mathrm{a}$ \\
\hline
\end{tabular}

$\mathrm{T} 1=40-40-00 ; \mathrm{T} 2=80-40-00 ; \mathrm{T} 3=120-40-00 ; \mathrm{T} 0=00-00-00$. Valores que comparten la misma letra en la misma columna son estadísticamente iguales (Tukey $p=0.05$ ). 
Con base en estos resultados, para efectuar las simulaciones con el RothC, se usó el valor

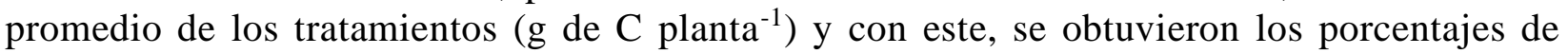
entrada C de RV de 60, 70 y $80 \%$ del a MST, estos últimos, fueron cercanos a los reportados por González et al. (2017) para maíz bajo labranza de conservación en la región de Bajío (2.6 a $5.9 \mathrm{Mg}$ de $\mathrm{C} \mathrm{ha} \mathrm{añ}^{-1}$ ).

En la comparación de medias de las variables de crecimiento y rendimiento por variedad de quinua en los tratamientos de fertilización, no hubo diferencias significativas (Cuadro 5), lo que se explica por el nivel de fertilidad favorable del suelo donde la quinua se estableció, con un contenido de materia orgánica del suelo de $2.3 \%$ y de nitrógeno mineral de $91 \mathrm{ppm}$, ya que la quinua es un cultivo que mantiene su productividad aun en suelos con baja fertilidad, poca humedad e incluso alta salinidad (Ruiz et al., 2014). Sobre la distribución del C por órgano en los tratamientos de fertilización (Figura 1).
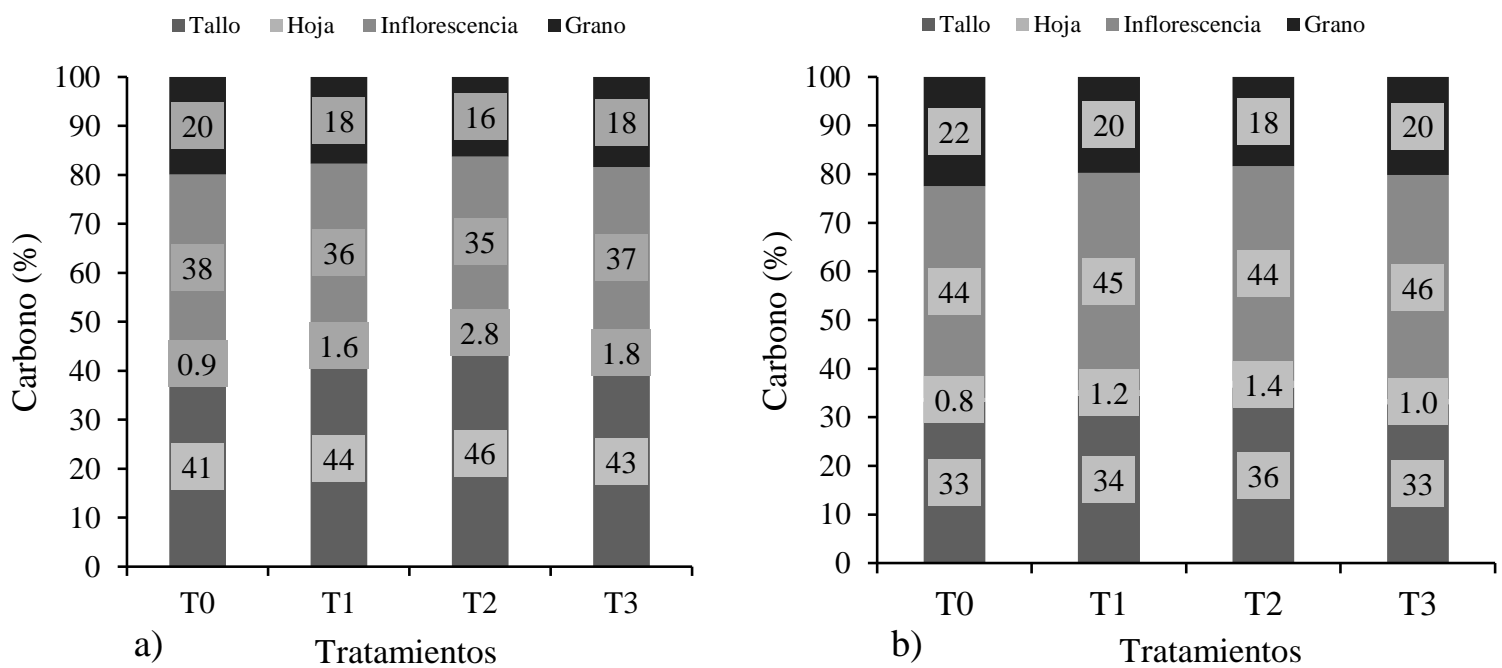

Figura 1. Distribución de carbono por estructura vegetal en los tratamientos de fertilización en madurez fisiológica en las quinuas; a) Amarilla Maranganí y b) Blanca. T1: 40-40-00; T2: 80-40-00; T3: 120-40-00; y T0: 00-00-00.

Se encontró que en ambas quinuas los tallos y las inflorescencias son cerca de un $80 \%$ de la MST. En Amarilla Maranganí es mayor el C en los tallos, 41-46\% de la MST, mientras que en Blanca es mayor en las inflorescencias, 44-46\% de MST. En ambas quinuas, las hojas y grano representan menor proporción de la MST, de un 0.8 a $2.8 \%$ y de 16 a $22 \%$ de la MST, respectivamente. Esto dio como resultado, bajo IC (0.17-0.18) que se refleja en alta producción de MST en hojas, tallos e inflorescencias y baja producción de MST en grano. Resultados que estuvieron cercanos con lo reportado por Mujica et al. (2003) para quinua, con 45\% para los tallos, 29\% para las hojas e inflorescencias y $26 \%$ para el grano.

\section{Simulación de escenarios de COS con diferentes aportes de RV}

Los resultados de simulación de escenarios con diferentes aportes $\mathrm{C}$ y en diferentes periodos de tiempo se presentan en el Cuadro 7 y Figura 2. En los periodos de tiempo evaluados, hubo secuestro de COS solo en MQ con el aporte de C al suelo de RV de 80\% de MST (0.02-0.1 Mg 
$\mathrm{C}$ ha $\left.{ }^{-1} \mathrm{año} \mathrm{o}^{-1}\right)$, mientras que, el orden de pérdidas de COS de menor a mayor fue el siguiente: MQ con RV de 70\% MTS< MQ con RV de 60\% MST (entre 0 a -0.16) < RQM (-0.02 a -0.18); y MM (entre -0.11 a -0.19 ).

Cuadro 7. Cambios de carbono orgánico del suelo a 20, 60 y 100 años de cultivo continuo en tres entradas de residuos vegetales en la profundidad de suelo $0-30 \mathrm{~cm}$.

\begin{tabular}{|c|c|c|c|c|c|c|c|}
\hline \multirow{3}{*}{$\begin{array}{c}\text { Año } \\
(\% \text { MST) }\end{array}$} & \multicolumn{3}{|c|}{ Monocultivo de quinua } & \multicolumn{3}{|c|}{ Rotación quinua + maíz } & \multirow{2}{*}{$\frac{\text { Monocultivo maíz }}{40}$} \\
\hline & 60 & 70 & 80 & $60+40$ & $70+40$ & $80+40$ & \\
\hline & \multicolumn{7}{|c|}{$\left(\mathrm{Mg} \mathrm{ha}^{-1} \mathrm{año}^{-1}\right)$} \\
\hline \multicolumn{8}{|c|}{ Amarilla Maranganí } \\
\hline 20 & -0.11 & -0.01 & 0.1 & -0.15 & -0.09 & -0.03 & -0.19 \\
\hline 40 & -0.08 & 0 & 0.07 & -0.11 & -0.07 & -0.02 & -0.14 \\
\hline 60 & -0.06 & 0 & 0.06 & -0.08 & -0.05 & -0.02 & -0.11 \\
\hline \multicolumn{8}{|c|}{ Blanca } \\
\hline 20 & -0.16 & -0.07 & 0.03 & -0.18 & -0.13 & -0.07 & -0.19 \\
\hline 40 & -0.12 & -0.05 & 0.02 & -0.13 & -0.09 & -0.05 & -0.14 \\
\hline 60 & -0.09 & -0.04 & 0.02 & -0.1 & -0.07 & -0.04 & -0.11 \\
\hline
\end{tabular}

MST= materia seca total.
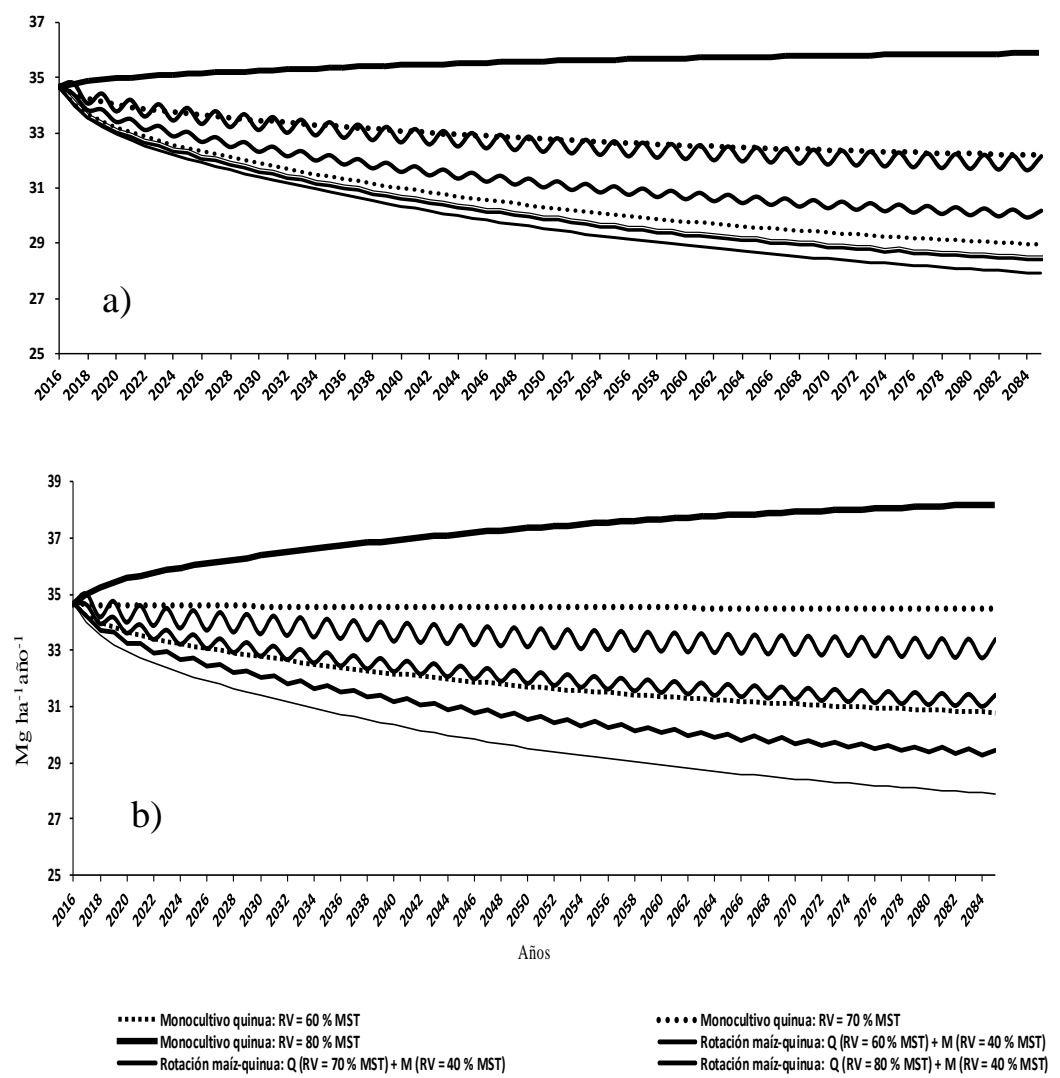

Figura 2. Escenarios de cambios de carbono orgánico del suelo con diferentes aportes de carbono al suelo en las quinuas: a) Blanca y b) Amarilla Maranganí. 
Esto puede explicarse por el tiempo de conversión o cambio del COS, que de acuerdo con Jenkinson y Rayner (1977), es el movimiento del C orgánico, a través de un volumen dado de suelo, y se obtiene de la relación entre el COS y la entrada anual de RV. El tiempo de conversión promedio (años) para los diferentes periodos de simulación con el RothC en los sistemas estudiados tuvo el orden inverso al COS secuestrado: MM (9.3)> RQM (8.5)> MQ con 40\% de MST (8.9)> MQ con $60 \%$ de MST (7.7)> MQ con $80 \%$ de MST (6.7).

Los valores más altos de conversión de COS: MM y RQM, indicaron la estabilización del C en el suelo, y los más bajos: MQ con $80 \%$ de MST, indicaron que hubo una migración más rápida del C y por lo tanto, indicaron que el suelo está más lejano del estado de equilibrio por una mayor entrada de RV (Figura 1, Cuadro 4). El COS almacenado o secuestrado estuvo dentro de lo reportado a escala mundial por Lal (2004), quien reporta de 0.02 a $0.76 \mathrm{Mg} \mathrm{ha}^{-1}$ año $^{-1}$ para el caso de sistemas agrícolas que adoptan sistemas de manejo mejorado.

En general, la quinua Amarilla Maranganí en monocultivo y bajo rotación con maíz superó las tasas de cambio de COS de la Blanca en monocultivo y de monocultivo de maíz con las entradas de $\mathrm{C}$ al suelo evaluadas con el RorhC, resultados que tuvieron una relación lineal con su mejor crecimiento, rendimiento y entradas de $\mathrm{C}$ al suelo con relación a Blanca. Las entradas de $\mathrm{C}$ al suelo por los RV evaluados tuvieron relación lineal con las tasas de secuestro (Cuadro 5 y Cuadro 6).

Similarmente a lo encontrado por Wang et al. (2017) quienes, a nivel global en cereales con diferentes entradas de $\mathrm{C}$ al suelo, tuvieron una correlación alta con los cambios del COS. Particularmente, las tasas de cambio de COS encontradas en quinua de nuestro trabajo respondieron a las condiciones particulares de la productividad de la materia seca influenciada por el clima y retención de residuos, aplicación de abonos y las salidas de MST, como reporta Smith et al. (2008); Luo et al. (2010) en los sistemas agrícolas. Las ejecuciones de escenarios con el RothC detectaron que ambas quinuas responden a las necesidades actuales del uso de cultivos con potencial de secuestro de COS y para mantener el suelo en una condición de equilibrio entre las ganancias y pérdidas de este elemento (Figura 2).

\section{Conclusiones}

La fertilización al suelo en quinua no tuvo efecto significativo en la acumulación de carbono en la materia seca por estructura vegetal y materia seca total, esto se atribuyó al nivel favorable de fertilidad del suelo donde se establecieron y a la rusticidad del cultivo. Amarilla Maranganí en monocultivo fue la de mayor secuestro de carbono orgánico del suelo con RV=80\% MST. Ambas quinuas mostraron mayor potencial de secuestro bajo condición de monocultivo que cultivadas en rotación con maíz y que el maíz en monocultivo, en periodos de tiempo mayores a 20 años.

\section{Literatura citada}

Bazile, D.; Bertero, D. y Nieto, C. 2014. Estado del arte de la quinua en el mundo en 2013: FAO (Santiago de Chile) y CIRAD, (Montpellier, Francia). 724 p.

Coleman, K.; Jenkinson, D. S.; Crocker, G. J.; Grace, P. R.; Klir, J.; Korschens, M.; Poulton, P. R. and Richter, D. D. 1997. Simulating trends in soil organic carbon in long-term experiments using RothC-26.3. Geoderma. 81(1-2):29-44. 
Coleman, K. and Jenkinson, D. 2005. ROTHC-26.3. A model for the turnover of carbon in soil. Model description and Windows Users' Guide. Rothamsted, U. K. Harpenden: Rothamsted Research. 1-43 pp.

Delatorre, H. J.; Salinas B. A. and Sánchez, M. M. 2008. The cultivation of quinoa. Universidad Arturo Prat Iquique. Chile. 135 p.

Falloon, P.; Smith, P.; Coleman, K. and Marshall, S. 1998. Estimating the size of the inert organic matter pool from total soil organic carbon content for use in the Rothamsted carbon model. Soil Bio. and Biochem. 30(8-9):1207-1211.

González, M. L.; Etchevers, B. J. D.; Paz, P. F.; Díaz Solís, H.; Fuentes, P. M. H.; Covaleda, O. S. and Pando, M. M. 2011. Performance of the RothC-26.3 model in short-term experiments in Mexican sites and systems. J. Agric. Sci. 149(4):415-425.

González, M. L.; Moreno, P. E. del C. and Baéz, P. A. 2017. Simulation of soil organic carbon changes in Vertisols under conservation tillage using the RothC model. Sci. Agr. 74(3):235-241.

INEGI (Instituto Nacional de Geografía e Informática). 2007. Conjunto de datos vectorial edafológico, Serie II, escala 1:250 000 (Continuo Nacional). México.

Jenkinson, D. S. and Rayner, J. H. 1977. The turnover of soil organic matter in some of the Rothamsted classical experiments. Soil Sci. 123(5):298-305

Kuzyakov, Y. and Domanski, G. 2000. Carbon input by plants into the soil. Review. J. Plant Nutrit. and Soil Sci. 163(4):421-431.

Lal, R. 2004. Soil carbon sequestration to mitigate climate change. Geoderma. 123(1-2):1-22.

Lal, R.; Follet, R. F.; Stewart, B. A. and Kimble, J. M. 2007. Soil carbon sequestration to mitigate climate change and advance food security. Soil Sci. 172(12):943-956.

Luo, Z.; Wang, E. and Sun, O. J. 2010. Soil carbon change and its responses to agricultural practices in Australian agro-ecosystems: A review and synthesis. Geoderma. 155(3-4):211-223.

Mujica, A.; Marca, S. and Jacobsen, S-E. 2003. Current production and potential of Chenopodium quinoa Willd.) in Peru. Food Rev. Int. 19(1-2):149-154.

Paustian, K.; Lehmann, J.; Ogle, S.; Reay, D.; Robertson, G. P. and Smith, P. 2016. Climate-smart soils. Nature. 532(7597):49-57.

Pérez, O. A.; Etchevers, J. D.; Navarro, G. H. y Nuñez, E. R. 2000. Aporte de los residuos del cultivo anterior al reservorio de nitrógeno en tepetates. Agrociencia. 34(2):115-125.

Smith, P. 2008. Land use change and soil organic carbon dynamics. Nut. Cycl. Agroecosyst. 81(2):169-178.

Ruiz, K. B.; Biondi, S.; Oses, R.; Acuna, R. I. S.; Antognoni, F.; Martínez, M. E. A.; Coulibaly, A.; Canahua, M. A.; Pinto, M.; Zurita, S. A. and Bazile, D. 2014. Quinoa biodiversity and sustainability for food security under climate change: a review. Agron. Sustain. Dev. 34(2):349-359.

SAGARPA (Secretaria de Agricultura, Ganadería, Desarrollo Rural, Pesca y Alimentación). 2015. Agenda Técnica Agrícola Estado de México. México. Segunda edición. México, DF. 288 p.

Walkley, A. and Black, I. A. 1934. An examination of the Degtjareff method for determining soil organic matter, and a proposed modification of the chromic acid titration method. Soil Sci. $37(1): 29-38$.

Wang G.; Zhang, W.; Sun, W.; Li, T. and Han, P. 2017. Modeling soil organic carbon dynamics and their driving factors in the main global cereal cropping systems. Atmos. Chem. and Phys. 17(19):11849-11859.

Zhang, W.; Yu, Y.; Li, T.; L.; Sun, W. and Huang, Y. 2014. Net greenhouse gas balance in China's Croplands over the last three decades and its mitigation potential. Environ. Sci. Tech. 48(5):2589-2597. 